Pacific Journal of Mathematics

THE DIRAC MONOPOLE AND INDUCED REPRESENTATIONS 


\title{
THE DIRAC MONOPOLE AND INDUCED REPRESENTATIONS
}

\author{
R. LANGLANDS
}

In this note a mathematically transparent treatment of the Dirac monopole is given from the point of view of induced representations. Among other things the question of bound states for the spinning electron in the field of a magnetic monopole is considered.

In 1948, as he was turning from theoretical physics to mathematics, Harish-Chandra wrote one last paper [5] on a physical topic, investigating a question inspired by Dirac, and perhaps even proposed by him. Does an electron moving according to Dirac's equation in the field of a magnetic monopole have a bound state? The technique involved, namely, separation of variables, is of course elementary, and would reappear but at a much deeper level repreatedly in Harish-Chandra's later work on harmonic analysis on semisimple groups. However, this time, his mind on other matters, he handled it perfunctorily, and went astray with the calculations, concluding - incorrectly - that there could be no bound states. The matter has since been dealt with correctly ([4], [6], [8]). However, the derivation of the radial equations in [6] and [8] is not so efficient as it might be. In view of the possible historical interest to students of Harish-Chandra's later work, a brief, mathematically transparent treatment does not seem out of place. It is the purpose of this note, which does not touch on any questions of serious current interest, to provide it.

In [2] Dirac considers the wave function $\psi$ of a charged particle without spin, observing that one can replace $\psi$ by $e^{i \beta} \psi$ without changing the distribution $|\psi|^{2}$, which is what counts. The function $\beta$ depends on the coordinates $t, x^{1}, x^{2}, x^{3}$. The substitution replaces

$$
\frac{1}{i} \frac{\partial}{\partial t} \text { by } \frac{1}{i} \frac{\partial}{\partial t}+\frac{\partial \beta}{\partial t}
$$

and

$$
\frac{1}{i} \frac{\partial}{\partial x^{j}} \text { by } \frac{1}{i} \frac{\partial}{\partial x^{j}}+\frac{\partial \beta}{\partial x^{j}}
$$

but, since

$$
\frac{1}{i} \frac{\partial}{\partial t} \text { and } \frac{1}{i} \frac{\partial}{\partial x^{j}}
$$


do not appear alone but in the combinations

$$
\frac{1}{i} \frac{\partial}{\partial t}+A_{0}, \quad \frac{1}{i} \frac{\partial}{\partial x^{j}}+A_{j}
$$

this effect can be countered by a change in the electromagnetic potentials $A_{0}, A_{1}, A_{2}, A_{3}$. The upshot is that $\psi$ can be regarded as a section of a line bundle with metric and with a connection defined by the electromagnetic potentials.

The pertinent line bundle is on $M$, four-dimensional space with the line $x^{1}=x^{2}=x^{3}=0$ removed. This space is to be identified with $\mathbf{R} \times \mathbf{R}^{+} \times H \backslash G$, where $G$ is $\mathrm{SU}(2)$ and $H$ is the group of diagonal matrices. As usual $G$ is mapped to $\mathrm{SO}(3)$ and thus acts on 3-space $\left(\left(x^{1}, x^{2}, x^{3}\right) \rightarrow\left(y^{1}, y^{2}, y^{3}\right)=\left(x^{1}, x^{2}, x^{3}\right) A(g)\right)$ in such a way that the Pauli matrices

$$
\sigma^{1}=\left(\begin{array}{ll}
0 & 1 \\
1 & 0
\end{array}\right), \quad \sigma^{2}=\left(\begin{array}{rr}
0 & -i \\
i & 0
\end{array}\right), \quad \sigma^{3}=\left(\begin{array}{rr}
1 & 0 \\
0 & -1
\end{array}\right)
$$

multiplied by $i / 2$ correspond to infinitesimal rotations through the $x^{1}, x^{2}$, and $x^{3}$-axes. Thus

$$
\left(\begin{array}{cc}
y^{3} & y^{1}-i y^{2} \\
y^{1}+i y^{2} & -y^{3}
\end{array}\right)=g^{-1}\left(\begin{array}{cc}
x^{3} & x^{1}-i x^{2} \\
x^{1}+i x^{2} & -x^{3}
\end{array}\right) g .
$$

Then $(r, g) \rightarrow r\left(\begin{array}{lll}0 & 0 & 1\end{array}\right) A(g)$ identifies $\mathbf{R}^{+} \times H \backslash G$ with 3 -space minus the origin. The line bundle is defined by a one-dimensional representation

$$
\rho:\left(\begin{array}{cc}
e^{i \theta} & 0 \\
0 & e^{-i \theta}
\end{array}\right) \rightarrow e^{i n \theta}
$$

of $H$, sections being complex functions $f$ on $\mathbf{R} \times \mathbf{R}^{+} \times G$ satisfying

$$
f(t, r, h g)=\rho(h) f(t, r, g)
$$

The connection, like the bundle, is a product, trivial on the first two factors. Let $\mathscr{H}^{\perp}$ be the orthogonal complement of $\mathscr{H}$ in $G$ and regard functions on $H \backslash G$ as $H$-invariant functions on $G$. We prescribe that for $X \in \mathscr{H}^{\perp}$ the tangent vector $X_{g}$ at $H g$ defined by

$$
X_{g} f=\left.\frac{d}{d t} f(\exp t X \cdot g)\right|_{t=0}
$$

acts on sections by the same formula, and verify that this yields a well-defined connection.

The curvature of this connection can be computed on $H \backslash G$ and, since $\left[\mathscr{H}^{\perp}, \mathscr{H}^{\perp}\right] \subseteq \mathscr{H}$, is a two-form that takes the value $\frac{1}{2} \rho\left(\left[X^{1}, X^{2}\right]\right)$ at $X_{g}^{1} \wedge X_{g}^{2}$. Taking $X^{1}=i \sigma^{1} / 2, X^{2}=i \sigma^{2} / 2$ and recalling that when divided by $-i / 2$ the curvature on $M$ yields a two-form that gives the electric 
and magnetic fields, we see that the associated electric field is 0 and the magnetic field purely radial and equal to

$$
-\frac{n}{2 r^{3}}\left(x^{1}, x^{2}, x^{3}\right) \text {. }
$$

Observe that all constructions are invariant under the action of $G$ on $H \backslash G$, which of course yields the usual action of $G$ on $M$. Consequently the magnetic field is spherically symmetric and need only be evaluated at $(0,0, r)$. Notice also that at a point $(r, g)$ where $g=1$,

$$
X_{1}^{1}=-r \frac{\partial}{\partial x^{2}}, \quad X_{1}^{2}=r \frac{\partial}{\partial x^{1}} .
$$

However, the Dirac equation, with which [5] was concerned, is for electrons with spin. To introduce it we tensor the bundle with the four-dimensional trivial bundle on $M$. If $\sigma$ is the representation of $G$ on four-dimensional space obtained by adding the identity representation of $G$ to itself, then the equations are invariant under the transformation $F \rightarrow F^{\prime}$ with

$$
(1 \otimes \sigma(g)) F^{\prime}(t, \vec{x} A(g))=F(t, \vec{x})
$$

So we replace $F$ by $f$ with

$$
f(t, r, g)=(1 \otimes \sigma(g)) F(t, r(0,0,1) A(g))
$$

to obtain equations invariant under right translations.

In terms of $f$ the Dirac operator may be written

$$
i\left(\gamma^{0} \frac{\partial}{\partial t}+\gamma^{3} \frac{\partial}{\partial r}+\frac{\gamma^{1}}{r} X^{2}-\frac{\gamma^{2}}{r} X^{1}+\frac{1}{r} \gamma^{3}\right)-m .
$$

The notation is that of [1]. This need only be verified at $(t, r, 1)$. Using equations (1) we see that the Dirac operator may be written as

$$
i(1 \otimes \sigma(g))\left(\gamma^{0} \frac{\partial}{\partial t}+\frac{\gamma^{1}}{r} X^{2}-\frac{\gamma^{2}}{r} X^{1}+\gamma^{3} \frac{\partial}{\partial r}\right)\left(1 \otimes \sigma\left(g^{-1}\right)\right)-m,
$$

$g$ being set equal to 1 after the differentiation. Thus it is

$$
i\left(\gamma^{0} \frac{\partial}{\partial t}+\gamma^{3} \frac{\partial}{\partial r}+\frac{\gamma^{1}}{r} X^{1}-\frac{\gamma^{2}}{r} X^{1}-\frac{\gamma^{1}}{r} \sigma\left(X^{2}\right)+\frac{\gamma^{2}}{r} \sigma\left(X^{1}\right)\right)-m .
$$

Since

$$
\gamma^{0}=\left(\begin{array}{rr}
1 & 0 \\
0 & -1
\end{array}\right), \quad \gamma^{j}=\left(\begin{array}{rr}
0 & \sigma^{j} \\
-\sigma^{j} & 0
\end{array}\right) \quad(j=1,2,3)
$$

and

$$
\sigma\left(X^{j}\right)=\frac{i}{2}\left(\begin{array}{cc}
\sigma^{j} & 0 \\
0 & \sigma^{j}
\end{array}\right)
$$

the equation (3) follows. 
The eigenfunction equation of the associated Hamiltonian is

$$
\begin{aligned}
\left\{\frac{1}{i}\left(\begin{array}{cc}
0 & \sigma^{3} \\
\sigma^{3} & 0
\end{array}\right) \frac{\partial}{\partial r}+\frac{1}{i}\left(\begin{array}{cc}
0 & \sigma^{1} \\
\sigma^{1} & 0
\end{array}\right) \frac{X^{2}}{r}\right. \\
\left.-\frac{1}{i}\left(\begin{array}{cc}
0 & \sigma^{2} \\
\sigma^{2} & 0
\end{array}\right) \frac{X^{1}}{r}+\frac{1}{i r}\left(\begin{array}{cc}
0 & \sigma^{3} \\
\sigma^{3} & 0
\end{array}\right)\right\} f \\
=\left(\begin{array}{cc}
E-m & 0 \\
0 & E+m
\end{array}\right) f .
\end{aligned}
$$

It cannot be interpreted until the formal expression on the left has been completely defined as an operator $H$ that will be an extension of the closure $H_{0}$ of the obviously defined operator on functions with compact support on $\mathbf{R}^{+} \times G$. Since $H_{0}$ will be seen not to be self-adjoint if $n \neq 0$, there will be some freedom in the choice of $H$.

To analyze $H_{0}$ and its self-adjoint extensions we can clearly consider the projections onto the space of functions $f$ transforming according to a given representation of $G$. Take the representation of dimension $d+1$ acting on the polynomials of degree $d$ in two variables with orthonormal basis $e_{j, k}=\left(\begin{array}{c}d \\ j\end{array}\right)^{1 / 2} x^{j} y^{k}, j+k=d$.

The entries of the column vector $f$ will then be matrix coefficients of the representation and we can take them all from one column, the several columns entailing a multiplicity. As a consequence of (2) the first and the third entries will be multiples $f_{1} / r$ and $f_{3} / r$ of the matrix coefficient on the row with $j-k=n+1$, and the second and fourth entries will be multiples $f_{2} / r$ and $f_{4} / r$ of the coefficient in the row with $j-k=n-1$.

The denominator simplifies the inner product

$$
\int_{\mathbf{R}^{+} \times G}\|f\|^{2} r^{2} d r d g=\sum_{i=1}^{4} \int_{0}^{\infty}\left|f_{i}(r)\right|^{2} d r
$$

and, when we rewrite (4) in terms of the $f_{i}$, removes the last term on the left. We have

$$
\sigma^{1} X^{2}-\sigma^{2} X^{1}=\left(\begin{array}{cc}
0 & X^{2}+i X^{1} \\
X^{2}-i X^{1} & 0
\end{array}\right)
$$

and, in the Lie algebra,

$$
\begin{aligned}
& X^{2}+i X^{1}=\frac{i}{2}\left(\sigma^{2}+i \sigma^{1}\right)=\left(\begin{array}{rr}
0 & 0 \\
-1 & 0
\end{array}\right), \\
& X^{2}-i X^{1}=\frac{i}{2}\left(\sigma^{2}-i \sigma^{1}\right)=\left(\begin{array}{ll}
0 & 1 \\
0 & 0
\end{array}\right) .
\end{aligned}
$$


Moreover

$$
\begin{aligned}
& \left(\begin{array}{ll}
0 & 0 \\
1 & 0
\end{array}\right): e_{j, k} \rightarrow(j(k+1))^{1 / 2} e_{j-1, k+1}, \\
& \left(\begin{array}{ll}
0 & 1 \\
0 & 0
\end{array}\right): e_{j, k} \rightarrow((j+1) k)^{1 / 2} e_{j+1, k-1} .
\end{aligned}
$$

So the left side of (4) becomes

and the right side

(6)

$$
\left(\begin{array}{cccc}
E-m & 0 & 0 & 0 \\
0 & E-m & 0 & 0 \\
0 & 0 & E+m & 0 \\
0 & 0 & 0 & E+m
\end{array}\right)\left(\begin{array}{c}
f_{1} \\
f_{2} \\
f_{3} \\
f_{4}
\end{array}\right)
$$

Here $\mu=((j+1) k)^{1 / 2}=\sqrt{(d+1)^{2}-n^{2}} / 2, j+k=d, j-k=n-$ 1 . In the special case $d+1=-n$, the coefficients $f_{2}$ and $f_{4}$ are fictitious, $\mu=0$, and (5) becomes

$$
\frac{1}{i}\left(\begin{array}{ll}
0 & 1 \\
1 & 0
\end{array}\right) \frac{\partial}{\partial r}\left(\begin{array}{l}
f_{1} \\
f_{3}
\end{array}\right) .
$$

If $d+1=n$ then (5) becomes

$$
-\frac{1}{i}\left(\begin{array}{ll}
0 & 1 \\
1 & 0
\end{array}\right) \frac{\partial}{\partial r}\left(\begin{array}{l}
f_{2} \\
f_{4}
\end{array}\right) .
$$

Taking

$$
\left(\begin{array}{l}
f_{1} \\
f_{2} \\
f_{3} \\
f_{4}
\end{array}\right)=\left(\begin{array}{c}
f \\
\varepsilon f \\
g \\
-\varepsilon g
\end{array}\right)
$$

with $\varepsilon= \pm 1$, we decouple the system given by (5) and (6) into the systems

(9) $\quad\left\{\frac{1}{i}\left(\begin{array}{ll}0 & 1 \\ 1 & 0\end{array}\right) \frac{\partial}{\partial r}+\frac{\varepsilon \mu}{i r}\left(\begin{array}{rr}0 & 1 \\ -1 & 0\end{array}\right)\right\}\left(\begin{array}{l}f \\ g\end{array}\right)=\left(\begin{array}{cc}E-m & 0 \\ 0 & E+m\end{array}\right)\left(\begin{array}{l}f \\ g\end{array}\right)$. 
In the two exceptional cases we have the system

$$
\pm \frac{1}{i}\left(\begin{array}{ll}
0 & 1 \\
1 & 0
\end{array}\right) \frac{\partial}{\partial r}\left(\begin{array}{l}
f \\
g
\end{array}\right)=\left(\begin{array}{cc}
E-m & 0 \\
0 & E+m
\end{array}\right)\left(\begin{array}{l}
f \\
g
\end{array}\right)
$$

The operators appearing on the left of (9) and (10) have still to be defined. They are to be self-adjoint extensions of the closure $T_{0}$ of the obviously defined operators on smooth functions of compact support on $(0, \infty)$. We apply the theory of [3], XIII. 2, which obviously extends to systems. Denote the formal differential operator on the left of (9) or (10) by $\tau$.

For equation (9) the roots of the indicial equation of $\tau$ at 0 are $\pm \mu$. Since $\mu \geq 1$, only one solution of

$$
\tau\left(\begin{array}{l}
f \\
g
\end{array}\right)= \pm i\left(\begin{array}{l}
f \\
g
\end{array}\right)
$$

is square-integrable on $(0,1]$, and there are no boundary conditions ([3], XII.4.21, XIII.2.19, XIII.2.23). For equation (10) the indicial equation has the multiple root 0 at 0 . So there are two independent boundary values. For both equations there is one square-integrable solution of (11) on $[1, \infty)$ and no boundary condition at $\infty$.

We conclude that for (9) the operator $T_{0}$ is already self-adjoint. It follows from Lemma XIII.4.23 of [3] and integration by parts that for (10)

$$
-i\left(T_{0}^{*} F, F^{\prime}\right)+i\left(F, T_{0}^{*} F^{\prime}\right)=f \bar{g}^{\prime}+g \bar{f}^{\prime},
$$

with $F=(f, g)^{t}, F^{\prime}=\left(f^{\prime}, g^{\prime}\right)^{t}$. So the self-adjoint extensions $T$ of $T_{0}$ are defined by $f(0)=i \lambda g(0), \lambda \in \mathbf{R}$, or $g(0)=0$.

In all cases the discrete spectrum of

$$
T+\left(\begin{array}{rrrr}
m & 0 & 0 & 0 \\
0 & m & 0 & 0 \\
0 & 0 & -m & 0 \\
0 & 0 & 0 & -m
\end{array}\right)
$$

is obtained by explicitly solving (9) or (10). For a square integrable solution $E$ must be real. For (10) there is a square-integrable solution of the equation only if $m^{2}>E^{2}$ and it is

$$
f=e^{-r \sqrt{m^{2}-E^{2}}}, \quad g=\frac{\mp 1}{i} \sqrt{\frac{m-E}{m+E}} e^{-r \sqrt{m^{2}-E^{2}}} .
$$

It satisfies the boundary conditions if and only if $-\sqrt{(m+E) /(m-E)}$ $=\lambda$. So there is a bound state if $-\infty<\lambda<0$ and none otherwise.

For (9) there could be a solution square-integrable at $\infty$ only if $m^{2}>E^{2}$ and then it would have to be a multiple of the pair with $f$ equal 
to the Whittaker function $W_{0, \nu}(\alpha r)$ ([7]) where $\alpha=2 \sqrt{m^{2}-E^{3}}, \nu=$ $\mu-\frac{1}{2}, \varepsilon>0, \nu=\mu+\frac{1}{2}, \varepsilon<0$. This pair cannot be square-integrable near 0 .

We can sum the discussion up with a theorem (cf. [4]).

THEOREM. If $n=0$ the operator $H_{0}$ is self-adjoint. If $|n|>0$ then there is a 1-parameter family of spherically symmetric self-adjoint extensions $H$ of $H_{0}$, parametrized by $\lambda \in \mathbf{R} \cup\{\infty\}$ and no others. The operator

$$
H+\left(\begin{array}{cccc}
m & 0 & 0 & 0 \\
0 & m & 0 & 0 \\
0 & 0 & -m & 0 \\
0 & 0 & 0 & -m
\end{array}\right)
$$

has a discrete spectrum if and only if $\lambda \in(-\infty, 0)$, and then it consists of the eigenvalue $E=m\left(\lambda^{2}-1\right)\left(1+\lambda^{2}\right)^{-1}$ with multiplicity $|n|$.

\section{REFERENCES}

[1] J. D. Bjorken and S. D. Drell, Relativistic Quantum Mechanics, (1964).

[2] P. A. M. Dirac, Quantised singularities in the electromagnetic field, Roy. Soc. London, Proc., A 133 (1931).

[3] N. Dunford and J. Schwartz, Linear Operators, Part II (1963).

[4] A. S. Goldhaber, Dirac particle in a magnetic field: Symmetries and their breaking by monopole singularities, Phys. Rev. D, 16, No. 6 (1977).

[5] Harish-Chandra, Motion of an electron in the field of a magnetic pole, Phys. Rev., 74 (1948).

[6] Y. Kazama, C. N. Yang, and A. S. Goldhaber, Scattering of a Dirac particle with charge ze by a fixed magnetic monopole, Phys. Rev. D 15, No. 8 (1977).

[7] E. T. Whittaker, An expression of certain known functions as generalized hypergeometric functions, Bull. Amer. Math. Soc., 10 (1903).

[8] T. T. Wu and C. N. Yang, Dirac monopole without strings: Monopole harmonics, Nucl. Phys. B., 107 (1976).

Received October 16, 1985.

INSTITUTE FOR ADVANCED STUDY

Princeton, NJ 08540 



\section{PACIFIC JOURNAL OF MATHEMATICS EDITORS}

\author{
V. S. VARADARAJAN \\ (Managing Editor) \\ University of California \\ Los Angeles, CA 90024 \\ Herbert Clemens \\ University of Utah \\ Salt Lake City, UT 84112 \\ R. FINN \\ Stanford University \\ Stanford, CA 94305
}

\section{HERMANN FLASCHKA}

University of Arizona

Tucson, AZ 85721

RAMESH A. GANGOLLI

University of Washington

Seattle, WA 98195

VAUGHAN F. R. JONES

University of California

Berkeley, CA 94720

ROBION KIRBY

University of California

Berkeley, CA 94720
C. C. MOORE

University of California

Berkeley, CA 94720

H. SAMELSON

Stanford University

Stanford, CA 94305

HAROLD STARK

University of California, San Diego

La Jolla, CA 92093

\section{ASSOCIATE EDITORS}
R. ARENS
E. F. BECKENBACH
B. H. NEUMANN
F. WOLF
K. YOSHIDA
(1906-1982)

\section{SUPPORTING INSTITUTIONS}

UNIVERSITY OF ARIZONA UNIVERSITY OF OREGON

UNIVERSITY OF BRITISH COLUMBIA UNIVERSITY OF SOUTHERN CALIFORNIA

CALIFORNIA INSTITUTE OF TECHNOLOGY STANFORD UNIVERSITY

UNIVERSITY OF CALIFORNIA

MONTANA STATE UNIVERSITY

UNIVERSITY OF HAWAII

UNIVERSITY OF NEVADA, RENO

UNIVERSITY OF TOKYO

NEW MEXICO STATE UNIVERSITY

UNIVERSITY OF UTAH

OREGON STATE UNIVERSITY

WASHINGTON STATE UNIVERSITY

UNIVERSITY OF WASHINGTON 


\section{Pacific Journal of Mathematics}

\section{Vol. 126, No. $1 \quad$ November, 1987}

John Dauns, Uniform dimensions and subdirect products $\ldots \ldots \ldots \ldots \ldots 1$

William B. Jacob, Quadratic forms over dyadic valued fields. I. The graded

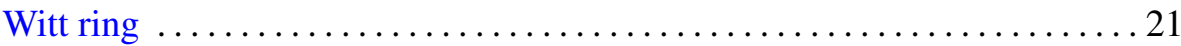

Michael R. Kelly, Minimizing the number of fixed points for self-maps of

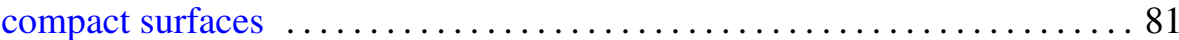

Edward Kissin, On some reflexive operator algebras constructed from two sets of closed operators and from a set of reflexive operator algebras . . 125

Robert Langlands, The Dirac monopole and induced representations . . . . . 145

David A. Stegenga and Kenneth R. Stephenson, Generic covering properties for spaces of analytic functions. II $\ldots \ldots \ldots \ldots \ldots \ldots \ldots 153$

Gerard Alan Venema, Approximating codimension two embeddings of cells 165

Peter Waksman, Determining an analytic function from its distribution of values 